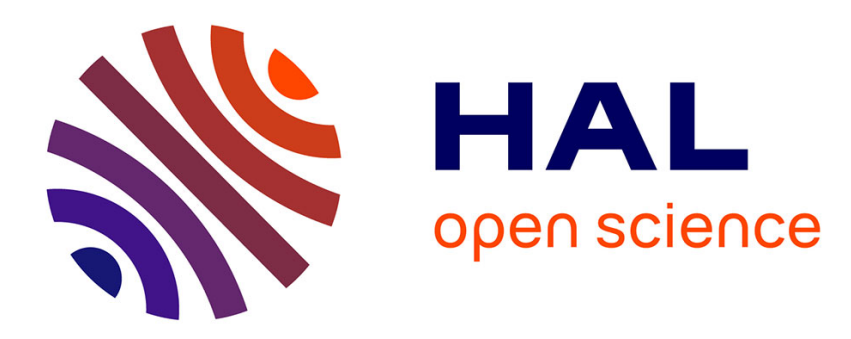

\title{
Continuity of the SRB Entropy of Convex Projective Structures
}

\author{
Patrick Foulon, Inkang Kim
}

\section{To cite this version:}

Patrick Foulon, Inkang Kim. Continuity of the SRB Entropy of Convex Projective Structures. 2021. hal-03132141

\section{HAL Id: hal-03132141 \\ https://hal.science/hal-03132141}

Preprint submitted on 4 Feb 2021

HAL is a multi-disciplinary open access archive for the deposit and dissemination of scientific research documents, whether they are published or not. The documents may come from teaching and research institutions in France or abroad, or from public or private research centers.
L'archive ouverte pluridisciplinaire HAL, est destinée au dépôt et à la diffusion de documents scientifiques de niveau recherche, publiés ou non, émanant des établissements d'enseignement et de recherche français ou étrangers, des laboratoires publics ou privés. 


\title{
CONTINUITY OF THE SRB ENTROPY OF CONVEX PROJECTIVE STRUCTURES
}

\author{
PATRICK FOULON AND INKANG KIM
}

\begin{abstract}
The space of convex projective structures has been well studied with respect to the topological entropy. But, to better understand the geometry of the structure, we study the entropy of the Sinai-Ruelle-Bowen measure and show that it is a continuous function on the space of strictly convex real projective structures.

\section{in memoriam Rufus Bowen}

\section{INTRODUCTION}

A topological manifold $M$ can be equipped with a $(G, X)$-structure where $X$ is a model space and $G$ is a group acting on $X$, so that $M$ has an atlas $\left\{\left(\phi_{i}, U_{i}\right)\right\}$ from open sets $\left\{U_{i}\right\}$ in $M$ into open sets in $X$ and the transition maps $\left\{\phi_{i} \circ \phi_{j}^{-1}\right\}$ are restrictions of elements in $G$. Depending on the choice of $(G, X)$, many interesting geometric structures can arise. For instance, if $M$ is a closed surface with genus at least 2, a hyperbolic structure corresponds to $\left(\operatorname{PSL}(2, \mathbb{R}), \mathbb{H}^{2}\right)$, a real projective structure to $\left(\mathrm{PGL}(3, \mathbb{R}), \mathbb{R P}^{2}\right)$, and a complex projective structure to $\left(\operatorname{PSL}(2, \mathbb{C}), \mathbb{C P}^{1}\right)$.

Given a geometric structure, there exist a developing map $D: \widetilde{M} \rightarrow X$ which is a local homeomorphism and a holonomy representation $\rho$ : $\pi_{1}(M) \rightarrow G$, so that $D$ is $\rho$-equivariant. In this paper, we study the real projective structures $\left(\operatorname{PGL}(n+1, \mathbb{R}), \mathbb{R} \mathbb{P}^{n}\right)$, and especially the strictly convex real projective structures $\mathcal{P}_{n}(M)$ on a closed manifold $M$, that is when $\Omega=D(\widetilde{M})$ is a strictly convex open domain of $\mathbb{R} \mathbb{P}^{n}$.

The set $\mathcal{P}_{n}(M)$ of equivalence classes of such strictly convex real projective structures, in the case of a closed surface, is known as Hitchin component in a character variety [15, 25]. It has drawn much attention

\footnotetext{
${ }^{1} 2000$ Mathematics Subject Classification. 51M10, 57S25.

${ }^{2}$ Key words and phrases. Real projective structure, Sinai-Ruelle-Bowen measure, entropy.

${ }^{3}$ I. Kim gratefully acknowledges the partial support of Grant (NRF2019R1A2C1083865) and KIAS Individual Grant (MG031408), and the warm support of CIRM during his visit.
} 
recently and many aspects of the set have been studied. The space has been identified as the holomorphic vector bundle over Teichmüller space [5, 13, 14, 29, 33, using affine sphere theory. It is also identified with the space of Anosov representations [30]. Explicit coordinates are studied [8, 9, 21, 24] and the space has a mapping class group invariant Kähler metric [26]. In this paper, we want to address the dynamical aspects related to the geodesic flow on the tangent bundle in any dimension. Crampon developed many aspects of this point of view in his papers [17, 18, 19, 20]. There are several measures invariant under the geodesic flow, but we single out one measure, the Sinai-RuelleBowen measure, abbreviated SRB measure. That measure provides, especially in dimension two, some deep insight concerning the shape of the boundary at infinity. We will elaborate on this in the text.

The most studied invariant measure is the Bowen-Margulis measure. A key property used by Crampon is that this measure is reversible, i.e., it is invariant under the flip map $\sigma(x,[v])=(x,[-v])$. The most eminent consequence is that the sum of the positive Lyapunov exponents associated to that measure is $n-1$, as in the hyperbolic metric case.

Using the Ruelle inequality for the Bowen-Margulis measure, Crampon deduces that the topological entropy is less than or equal to $n-1$ with equality only in the hyperbolic case. One key feature of the SRB measure is that the Ruelle inequality becomes an equality, known as the Pesin formula. When there is an invariant volume form, that form gives, after normalization, the density of the SRB measure. But we know from Benoist [4] that if the convex structure is not the hyperbolic model, then there is not such an invariant volume form for the flow. Nevertheless, in general the SRB measure is characterised by the fact that its conditional measures on unstable leaves are absolutely continuous to the Lebesgue measure (Ledrappier-Young [32]). This is why it turns out to be a key ingredient to better understand the geometry of projective structures. We also observe a global irreversible effect along the orientation of time. We will elaborate on this in Corollary 1.2 (3).

Theorem 1.1. Let $M$ be a closed manifold of dimension $n$. Then the map $h_{S R B}: \mathcal{P}_{n}(M) \rightarrow \mathbb{R}$ is continuous, where $h_{S R B}$ denotes the SinaiRuelle-Bowen measure entropy of the geodesic flow defined on the convex real projective manifold, and $\mathcal{P}_{n}(M)$ is the space of strictly convex real projective structures on $M$.

Given a strictly convex real projective manifold $M$ and the geodesic flow invariant SRB measure $\mu_{S R B}$, by ergodicity of the SRB measure, there exists a set $W_{S R B} \subset H M=(T M \backslash\{0\}) / \mathbb{R}_{+}^{*}$ with $\mu_{S R B}\left(W_{S R B}\right)=$ 
1 , and $\chi_{1}, \cdots, \chi_{p} \in \mathbb{R}$ called the Lyapunov exponents relative to the SRB measure, such that, for any $w \in W_{S R B}$, there exists a geodesic flow invariant decomposition $T H M=\oplus E_{i}$ and for any $v \in E_{i}, \chi_{i}(w, v)=$ $\chi_{i}$, where $\chi_{i}(w, v)$ are Lyapunov exponents. See Sections 2 and 2.2 for definitions and properties.

Corollary 1.2. Let $M_{t}$ be a smooth family of real projective structures on $M$. Then

(1) The sum $\chi^{+}$of the SRB positive Lyapunov exponents vary continuously in $t$.

(2) The entropy $h_{S R B}$ of the SRB measure satisfies inequality

$$
h_{S R B}=n-1+\eta \leq n-1
$$

where $\eta$ is the $S R B$ almost sure value of $\eta(w)=\sum \eta_{i}(w) \operatorname{dim} E_{i}^{u}(w)$, the sum of parallel Lyapunov exponents. By Ruelle inequality, $\eta \leq 0$ and the equality holds if and only if the structure is hyperbolic.

(3) (Irreversibility) Let dvol be any continuous volume form. Then

$$
\lim _{s \rightarrow \infty} \frac{1}{s} \log \left(\frac{d\left(\phi^{s}\right)^{*}(d v o l)}{d v o l}\right)=\sum \eta_{i} \operatorname{dim} E_{i}=2 \eta .
$$

As shown by Crampon [20], the Lyapunov exponents have to do with the convexity of the boundary of $\Omega$. The boundary $\partial \Omega$ in the neighborood of a point $p$ can be written as the graph of a $C^{1}$ strictly convex real function $f$ defined on $U$ open convex subset of $\mathbb{R}^{n-1}$. Such a function $f$ is said to be approximately $\alpha$-regular at $x_{0} \in \mathbb{R}^{n-1}$ if for all $v \in \mathbb{R}^{n-1}$, the limit

$$
\lim _{t \rightarrow 0} \frac{\log \left(f_{x_{0}}(t v)+f_{x_{0}}(-t v)\right)}{\log |t|}
$$

exists, where $f_{x_{0}}(v)=f\left(x_{0}+v\right)-f\left(x_{0}\right)-d_{x_{0}} f(v)$. Theorem 1.2 in [20] states that a function is approximately regular at the point $x_{0} \in U$ if and only if there exist an integer $1 \leq k \leq n-1$, a splitting $\mathbb{R}^{n-1}=$ $\oplus_{i=1}^{n-1} G_{i}$ and numbers $+\infty \geq \alpha_{1}\left(x_{0}\right)>\ldots \ldots .>\alpha_{p}\left(x_{0}\right) \geq 1$ such that for all $v \in G_{i}$

$$
\lim _{t \rightarrow 0} \frac{\log \left(f_{x_{0}}(t v)+f_{x_{0}}(-t v)\right)}{\log |t|}=\alpha_{i}\left(x_{0}\right) .
$$

In the sequel we will rather consider those exponents with their multiplicities and do the same for the positive Liapunov exponents. The link with the positive Lyapunov exponents is revealed in theorem 4.5 in $[20]$. Let $\Omega \subset \mathbb{R P}^{n}$ be a strictly convex set with $C^{1}$ boundary, a point $w \in H \Omega$ is weakly forward regular if and only if the boundary 
point $x^{+} \in \partial \Omega$ of the associated oriented line is approximately regular and for all $1 \leq i \leq n-1$,

$$
\alpha_{i}\left(x_{+}\right) \chi_{i}^{+}(w)=2 .
$$

Those quantities are invariant under affine and projective transformations. In dimension 2, approximately $\alpha$-regular means if $\alpha<\infty$ that the function behaves like $|t|^{\alpha}$ near the origin.

Corollary 1.3. There is a set $F_{S R B} \subset \partial \Omega$ of full Lebesgue measure and positive real numbers $1 \leq \alpha_{i}^{S R B}<\infty, 1 \leq i \leq n-1$, called the regular exponents for the boundary, such that for any $p \in F_{S R B}$ we have

$$
\alpha_{i}(p)=\alpha_{i}^{S R B} \text {. }
$$

Let $\alpha_{S R B}=(n-1)\left(\sum_{i=1}^{n-1} 1 / \alpha_{i}\right)^{-1}$ be their harmonic mean. Then the map $\alpha_{S R B}: \mathcal{P}_{n}(M) \rightarrow \mathbb{R}$ is continuous. Furthermore, we have

$$
\chi^{+} \alpha_{S R B}=2(n-1)
$$

and $\alpha_{S R B} \geq 2$ with equality if and only if the structure is hyperbolic.

Remarks. This shows that if a strictly convex projective structure is not the standard hyperbolic structure then the boundary is "very" flat. In particular for almost every point $p$ on the boundary the local representing graph admits at least a second derivative at $p$ that vanishes. But the boundary is nowhere $C^{2}$ [6, 28]. This remark enlights the work of Benoist [4], showing that the curvature of the boundary is localized on a set of null Lebesgue measure.

Acknowledgement. We thank F. Ledrappier for numerous discussions.

\section{PRELIMINARIES}

2.1. Projective structure. Let $M$ be an $n$-dimensional closed manifold equipped with a strictly convex projective structure so that $D$ : $\widetilde{M} \rightarrow \mathbb{R} \mathbb{P}^{n}$ is a developing map with $D(\widetilde{M})=\Omega$ and $\rho: \pi_{1}(M) \rightarrow \operatorname{PGL}(n+$ $1, \mathbb{R})$ the holonomy representation. Then it inherits a Hilbert metric defined on $\Omega$ in the following way. More precisely, for $x \neq y \in \Omega$, let $p, q$ be the intersection points of the line $\overline{x y}$ with $\partial \Omega$ such that $p, x, y, q$ are in this order. The Hilbert distance is defined by

$$
d_{\Omega}(x, y)=\frac{1}{2} \log \frac{|p-y||q-x|}{|p-x||q-y|}
$$

where $|\cdot|$ is a Euclidean norm in an affine chart containing $\Omega$. This metric coincides with the hyperbolic metric if $\partial \Omega$ is a conic. The Hilbert 
metric is Finsler rather than Riemannian. The Finsler norm $F=\|\cdot\|$ is given, for $x \in \Omega$ and a vector $v$ at $x$, by

$$
\|v\|_{x}=\left(\frac{1}{\left|x-p^{-}\right|}+\frac{1}{\left|x-p^{+}\right|}\right)|v|
$$

where $p^{ \pm}$are the intersection points of the line with $\partial \Omega$, defined by $x$ and $v$ with the obvious orientation, and where $|\cdot|$ is again a Euclidean norm. The Hilbert distance is invariant by projective transformations preserving $\Omega$ and thus the Finsler metric descends to the base manifold $M$. The induced Hilbert distance on $M$ also depends on the boundary of $\Omega$.

Also it is known that $\partial \Omega$ is $C^{1+\alpha}, \Gamma$ is Gromov hyperbolic and the geometry behaves like a negatively curved case. See [4, 6]. Since $\left(\Omega, d_{\Omega}\right)$ is $\delta$-hyperbolic [4], one can define the Busemann function as usual as

$$
b_{\xi}(x, y)=\lim _{t \rightarrow \infty} d_{\Omega}(x, \gamma(t))-t,
$$

where $\gamma$ is the geodesic such that $\gamma(0)=y, \gamma(\infty)=\xi \in \partial \Omega$ [17]. The horosphere passing through $x \in \Omega$ and based at $\xi \in \Omega$ is the set

$$
\mathcal{H}_{\xi}(x)=\left\{y \in \Omega: b_{\xi}(x, y)=0\right\} .
$$

For a given $w=(x,[\xi]) \in H \Omega=(T \Omega \backslash\{0\}) / \mathbb{R}_{+}^{*}$, the unstable manifold $W^{s u}$ passing through $w$ is defined to be

$$
W^{s u}(w)=\left\{(y,[\phi]) \in H \Omega \mid \xi(-\infty)=\phi(-\infty), y \in \mathcal{H}_{\sigma w}\right\} .
$$

Here $\xi(-\infty)$ denotes $\gamma_{\xi}(-\infty)$ where $\gamma_{\xi}$ is the geodesic determined by $\xi$, and $\sigma w=(x,[-\xi])$ is a flip map, and $\mathcal{H}_{w}$ is the horosphere based at $\xi(\infty)$ passing through $x$. Similarly one can define a stable manifold

$$
W^{s s}(w)=\left\{(y,[\phi]) \in H \Omega \mid \xi(\infty)=\phi(\infty), y \in \mathcal{H}_{w}\right\} .
$$

These stable and unstable manifolds are $C^{1}$ if $\partial \Omega$ is $C^{1}$.

The tangent spaces of $W^{s u}$ and $W^{s s}$ form unstable and stable vector bundles in $T H \Omega$, i.e., along the geodesic flow, they expand or decay exponentially. All the objects defined above descend to the quotient manifold $M$ and it is known [4] that the geodesic flow on $H M$ is Anosov with invariant decomposition

$$
T H M=\mathbb{R} X \oplus E^{s} \oplus E^{u},
$$

where $X$ is the vector field generating the geodesic flow. Note that the geodesic flow on $H M$ is only $C^{1+\alpha}$ if $M$ is not hyperbolic.

\subsection{Lyapunov exponents and Sinai-Ruelle-Bowen measure.}


2.2.1. Lyapunov exponents. Let $\phi=\phi^{t}$ be a $C^{1}$ flow on a Riemannian manifold $W$. A point $w \in W$ is said to be regular if there exists a $\phi^{t}$-invariant decomposition

$$
T W=E_{1} \oplus \cdots \oplus E_{p}
$$

along $\phi^{t} w$ and real numbers

$$
\chi_{1}(w)<\cdots<\chi_{p}(w),
$$

such that, for any vector $Z_{i} \in E_{i} \backslash\{0\}$,

$$
\lim _{t \rightarrow \pm \infty} \frac{1}{t} \log \left\|d \phi^{t}\left(Z_{i}\right)\right\|=\chi_{i}(w)
$$

and

$$
\lim _{t \rightarrow \pm \infty} \frac{1}{t} \log \left|\operatorname{det} d \phi^{t}\right|=\sum_{i=1}^{p} \operatorname{dim} E_{i} \cdot \chi_{i}(w) .
$$

The numbers $\chi_{i}(w)$ associated with a regular point $w$ are called the Lyapunov exponents of the flow at $w$. Due to Oseledets' multiplicative ergodic theorem [35], the set of regular points has full measure.

Theorem 2.1. Let $\phi$ be a $C^{1}$ flow on a Riemannian manifold $W$ and $\mu a \phi^{t}$-invariant probability measure. If

$$
\left.\frac{d}{d t}\right|_{t=0} \log \left\|d \phi^{ \pm t}\right\| \in L^{1}(W, \mu)
$$

then the set of regular points has full measure.

2.3. In the case of convex projective structures. Let $M$ be a closed strictly convex real projective manifold. There exists an extended Finsler norm $\bar{F}$ on $H M=(T M \backslash\{0\}) / \mathbb{R}_{+}^{*}$ (see, [17] Sec 2.3) and the Lyapunov exponent is defined to be

$$
\lim _{t \rightarrow \pm \infty} \frac{1}{t} \log \bar{F}\left(d \phi^{t}\left(Z_{i}\right)\right)=\chi_{i}(w) .
$$

Let $\Omega$ be a strictly convex domain with $C^{1}$ boundary equipped with a Hilbert metric. There is a notion of parallel transport $T^{t}$ due to Foulon [22, 23], see also [18] (Sec 4.4) or [17] (Sec 3.2.2). The parallel Lyapunov exponent of $v \in T_{x} \Omega$ along $\phi^{t}(x,[\psi])$ is defined to be

$$
\eta((x,[\psi]), v)=\lim _{t \rightarrow \infty} \frac{1}{t} \log F\left(T^{t}(v)\right) .
$$

Then Crampon [19] showed that a point $w=(x,[\psi]) \in H \Omega$ is regular if and only if there exists a decomposition

$$
T_{x} \Omega=\mathbb{R} \psi \oplus E_{0}(w) \oplus\left(\oplus_{i=1}^{p} E_{i}(w)\right) \oplus E_{p+1}(w),
$$


and real numbers

$$
-1=\eta_{0}(w)<\eta_{1}(w)<\cdots<\eta_{p}(w)<\eta_{p+1}=1,
$$

such that for any $v_{i} \in E_{i}(w) \backslash\{0\}$,

$$
\begin{gathered}
\lim _{t \rightarrow \pm \infty} \frac{1}{t} \log F\left(T_{w}^{t}\left(v_{i}\right)\right)=\eta_{i}(w) \\
\lim _{t \rightarrow \pm \infty} \frac{1}{t} \log \left|\operatorname{det} T_{w}^{t}\right|=\sum_{i=0}^{p+1} \operatorname{dim} E_{i}(w) \eta_{i}(w):=2 \eta(w) .
\end{gathered}
$$

Here $E_{0}$ and $E_{p+1}$ could be zero. The choice of a factor 2 in the formula is explained in the sequel.

The relation between Lyapunov and parallel Lyapunov exponents is; for stable $Z^{s}$ and unstable $Z^{u}$ vectors in $T_{w} H \Omega$,

$$
\chi\left(Z^{s}\right)=-1+\eta\left(w, d \pi\left(Z^{s}\right)\right), \chi\left(Z^{u}\right)=1+\eta\left(w, d \pi\left(Z^{u}\right)\right),
$$

where $\pi: H \Omega \rightarrow \Omega$ is the projection. See Proposition 1 of [19] for details.

Remark 2.2. The reversibility of the geodesic flow induces for regular points a special property observed by Crampon namely

$$
\eta((x,[\psi]), v)=-\eta(\sigma(x,[\psi]), \sigma(v))
$$

where $\sigma$ is the usual flip map. This means that if a trajectory of the flow is regular then the two opposite boundaries at $\infty$ are strongly related.

Hence one can summarize these facts as:

$$
T H \Omega=\mathbb{R} X \oplus\left(\oplus_{i=0}^{p+1}\left(E_{i}^{s} \oplus E_{i}^{u}\right)\right)
$$

where $E_{i}^{s}=J^{X}\left(E_{i}^{u}\right)$ for some pseudo-complex structure $J^{X}$ and $\chi_{i}^{-}$is a Lyapunov exponent for $E_{i}^{s}, \chi_{i}^{+}$for $E_{i}^{u}, E_{i}=E_{i}^{s} \oplus E_{i}^{u}$

$$
\begin{gathered}
\chi_{i}^{+}=1+\eta_{i}, \chi_{i}^{-}=-1+\eta_{i}, \chi_{i}^{+}=\chi_{i}^{-}+2 \\
-2=\chi_{0}^{-}<\chi_{1}^{-}<\cdots<\chi_{p+1}^{-}=0=\chi_{0}^{+}<\chi_{1}^{+}<\cdots<\chi_{p+1}^{+}=2 .
\end{gathered}
$$

Note that

$$
\begin{aligned}
\chi^{+}=\sum \operatorname{dim} E_{i}^{u} \chi_{i}^{+}= & \sum \operatorname{dim} E_{i}^{u}\left(1+\eta_{i}\right)=\sum \operatorname{dim} E_{i}^{u}+\sum \operatorname{dim} E_{i}^{u} \eta_{i} \\
& =(n-1)+\sum \operatorname{dim} E_{i}^{u} \eta_{i} .
\end{aligned}
$$

But

Hence

$$
2 \eta=\sum \operatorname{dim} E_{i} \eta_{i}=2 \sum \operatorname{dim} E_{i}^{u} \eta_{i}
$$

$$
\chi^{+}=(n-1)+\eta \text {. }
$$


Furthermore, from (1) we obtain the following relations between the exponents of the parallel transport and the regular exponents of the boundary

$$
\begin{gathered}
\eta_{i}=\frac{2}{\alpha_{i}}-1 \\
\eta(w, v(w))=(n-1)\left(\frac{2}{\alpha(\xi)}-1\right)
\end{gathered}
$$

where $\alpha=(n-1)\left(\sum_{i=1}^{n-1} 1 / \alpha_{i}\right)^{-1}$ is the harmonic mean of the exponents $\alpha_{i}$.

Remark 2.3. According to the remark about reversibility, for all regular trajectory $(x,[\psi])$ we have

$$
\frac{1}{\alpha(\xi)}+\frac{1}{\alpha(\sigma(\xi))}=1
$$

where $\sigma(\xi)=\psi(-\infty)$.

Periodic orbits are regular trajectories, and all these quantities can be expressed via the representation. For the reader's convenience we provide only the formulas in dimension 2. Suppose $\gamma$ is a hyperbolic isometry whose eigenvalues are $\lambda_{1}>\lambda_{2}>\lambda_{3}$ and $\gamma^{+}=\psi(\infty)$. Then it is shown in Proposition 5.5 of [18] (or in Section 3.6 of [17]) that

$$
\eta(w, v(w))=-1+2 \frac{\log \frac{\lambda_{1}}{\lambda_{2}}}{\log \frac{\lambda_{1}}{\lambda_{3}}},
$$

hence

$$
\alpha\left(\gamma^{+}\right)^{-1}=\frac{\log \frac{\lambda_{1}}{\lambda_{2}}}{\log \frac{\lambda_{1}}{\lambda_{3}}} .
$$

2.3.1. Invariant measures. For a geodesic flow on $H \Omega$, the maximal entropy $h_{\mu}(\phi)$ of the probability measure $\mu$ is known to be realized at the Bowen-Margulis measure [18]. This entropy is equal to the topological entropy $h_{\text {top }}(\phi)$ of the geodesic flow $\phi[18$ and it is also equal to the exponential growth of the lengths of closed geodesics:

$$
\lim _{R \rightarrow \infty} \frac{\log \#\{[\gamma] \mid \ell(\gamma) \leq R\}}{R}
$$

This is again equal to the critical exponent of the associated Poincaré series. See [17] (Sec 5.1). It is proved by Crampon [18] that the entropy of Bowen-Margulis measure of strictly convex real projective structures on a closed $n$-manifold is between 0 and $n-1$. But there exists another invariant measure called Sinai-Ruelle-Bowen measure, abbreviated 
SRB measure. It is characterized as follows. First we recall a fundamental theorem known as Margulis-Ruelle inequality, see [37] and [17] (Sec 5.2.1):

Theorem 2.4. Let $M=\Omega / \Gamma$ be a strictly convex compact projective manifold. Let $\mu$ be a geodesic flow invariant probability measure on HM. Then

$$
h_{\mu}(\phi) \leq \int \chi^{+} d \mu
$$

where $\chi^{+}=\sum \operatorname{dim} E_{i} \cdot \chi_{i}^{+}$denotes the sum of positive Lyapunov exponents.

An invariant measure that achieves equality in Margulis-Ruelle inequality is called Sinai-Ruelle-Bowen measure [11]. We know from Benoist [4] that the geodesic flow of strictly convex projective structure on a closed manifold is Anosov, hence admits a unique SRB measure.

Another description of this measure $\mu_{S R B}$ is: there exists a set $V$ of full Lebesgue measure such that for each continuous function $f: M \rightarrow \mathbb{R}$ and for every $x \in V$,

$$
\lim _{T \rightarrow \infty} \frac{1}{T} \int_{0}^{T} f\left(\phi^{s}(x)\right) d s=\int f d \mu_{S R B} .
$$

Ledrappier-Young [32] proved the following characterization of a Sinai-Ruelle-Bowen measure, see [3] or [17] (Sec 5.2.2):

Theorem 2.5. Let $M$ be a compact, strictly convex real projective manifold. Then the geodesic flow invariant measure $\mu$ is the SRB measure if and only if it has absolutely continuous conditional measures on unstable manifolds.

2.4. Deformation of the representation. Ehresmann-Thurston described the deformation of geometric structures in terms of the deformation of developing maps. This theory is rigorously rephrased by Bergeron-Gelander [7].

Proposition 2.6. If $\rho_{t}: \pi_{1}(M) \rightarrow S L(n, \mathbb{R})$ is a smooth deformation of strictly convex real projective structures, then there exists a continuous associated deformation $D_{t}$ of the developing map such that its image $\Omega_{t}$ and the boundary $\partial \Omega_{t}$ in Hausdorff topology vary continuously, hence the Hilbert metric $d_{t}$ and the geodesic flow $\phi_{t}$ vary continuously. Furthermore the convexity forces also the continuity of $\partial \Omega_{t}$ in $C^{1}$-topology.

Proof. For any two points $x, y \in M$, and two lifts $\tilde{x}, \tilde{y} \in \widetilde{M}$, we know that $D_{t} \tilde{x}, D_{t} \tilde{y}$ vary continuously according to Bergeron-Gelander. Then the line in $\mathbb{R P}^{n}$ connecting $D_{t} \tilde{x}, D_{t} \tilde{y}$ varies continuously. Now 
we need to determine two points on $\partial \Omega_{t}$ where this line intersects and show that they vary continuously. For each $\gamma \in \pi_{1}(M)$, let $\gamma_{t}^{+}$ be the line corresponding to the largest eigenvalue of $\rho_{t}(\gamma), \gamma_{t}^{-}$the line in $\mathbb{R}^{n+1}$ corresponding to the smallest eigenvalue, and $E_{t}^{\gamma}$ the sum of eigenspaces complementary to $\gamma_{t}^{-}$. Then it is known that $\gamma_{t}^{+} \in \partial \Omega_{t}$ and $T_{\gamma_{t}^{+}} \partial \Omega_{t}=E_{t}^{\gamma}$. Since $\rho_{t}$ varies continously, $\gamma_{t}^{+}$and $E_{t}^{\gamma}$ vary continuously. For any given finite set $\gamma_{1}, \cdots, \gamma_{k} \in \pi_{1}(M)$, $\Omega_{t}$ is included in the convex set formed by $\cap H E_{t}^{\gamma_{i}}$ where $H E_{t}^{\gamma_{i}}$ is the half space containing $\Omega_{t}$. By enlarging the set, we can see that $\Omega_{t}$ and $\partial \Omega_{t}$ vary continuously. More precisely, choose an increasing sequence of finite sets $\Gamma_{i} \subset \Gamma_{i+1} \subset \pi_{1}(M)$ such that $\cup \Gamma_{i}=\pi_{1}(M)$. Let $\Omega_{t} \subset \Omega_{t}^{i+1} \subset \Omega_{t}^{i}=\cap_{\gamma \in \Gamma_{i}} H E_{t}^{\gamma}$. Since $\left\{\gamma_{t}^{+}: \gamma \in \pi_{1}(M)\right\}$ is dense in $\partial \Omega_{t}$, $\Omega_{t}^{i} \rightarrow \Omega_{t}$ in Hausdorff topology as $i \rightarrow \infty$. Since $\gamma_{t}^{+}$and $E_{t}^{\gamma}$ vary continously in $t, \Omega_{t}^{i} \rightarrow \Omega_{t_{0}}^{i}$ for each fixed $i$ as $t \rightarrow t_{0}$. Then by taking diagonal sequence

$$
\Omega_{t}^{i_{n}} \rightarrow \Omega_{t_{0}}
$$

as $i_{n} \rightarrow \infty$ and $t \rightarrow t_{0}$.

Note that each point in $\mathcal{P}_{n}(M)$ is a class of $(D, \rho)$ where $D: \widetilde{M} \rightarrow \mathbb{R} \mathbb{P}^{n}$ is a developing map and $\rho: \pi_{1}(M) \rightarrow P G L(n+1, \mathbb{R})$ is a holonomy

representation. To each such pair $(D, \rho)$ are associated $\Omega_{D}=D(\widetilde{M})$, the Hilbert metric and the geodesic flow, hence $h_{S R B}^{D}$ the SRB entropy. If two structures $(D, \rho)$ and $\left(D^{\prime}, \rho^{\prime}\right)$ are equivalent, then $\rho$ and $\rho^{\prime}$ are conjugate and $\Omega_{D}$ and $\Omega_{D^{\prime}}$ are diffeomorphic by a projective map. Since the entropy is invariant under conjugacy, we have a well-defined map $h_{S R B}: \mathcal{P}_{n}(M) \rightarrow \mathbb{R}$. It is known that $\mathcal{P}_{n}(M)$ is a component of a character variety by Benoist [4]. Hence it is enough to study the continuity along a continuous path.

\section{Continuity of the Sinai-Ruelle-Bowen measure ENTROPY AND APPLICATION}

Throughout this section $M$ is a compact smooth manifold, $D$ : $\widetilde{M} \rightarrow \mathbb{R} \mathbb{P}^{n}$ is a developing map, and $\rho: \pi_{1}(M) \rightarrow P S L(n+1, \mathbb{R})$ is the holonomy map of a strictly convex real projective structure. Let $g^{h}$ be the Hilbert metric of $\Omega=D(\widetilde{M}), F$ the Hilbert norm on $T M$, and $\phi^{t}$ the geodesic flow associated with the Hilbert metric. Then it is known that this flow is Anosov and it is $C^{1, \alpha}$ due to Benoist 4 .

Let $g$ be a $C^{\infty}$-Riemannian metric on $M$. Suppose $\psi^{t}$ is a flow with the same trajectory as $\phi^{t}$ but unit speed with respect to $g$. If

$$
X=\frac{d \phi^{t}}{d t}, Z=\frac{d \psi^{t}}{d t},
$$


then

$$
X=m Z \text {. }
$$

We know that

$$
F(d \pi X)=g(d \pi Z)=1
$$

where $\pi: H M \rightarrow M$ is a projection. Then

$$
1=m(x,[v]) F(d \pi(Z(x,[v])),
$$

and set

$$
\beta=m^{-1}=F(d \pi Z)
$$

Lemma 3.1. $\psi^{t}$ and $Z$ are $C^{\infty}$ and $\phi^{t}$ and $X$ are $C^{1, \alpha}$. Furthermore $\beta: H M \rightarrow \mathbb{R}^{+}$is $C^{1, \alpha}$.

Proof. The geodesic foliation of a strictly convex projective structure is smooth because locally it is the foliation by straight lines in a projective chart. This was previously observed by Benoist. The fact that $\phi^{t}$ is $C^{1, \alpha}$ is also due to Benoist. The $C^{1}$-ness comes from the $C^{1}$-ness of Finsler norm, which is again due to the $C^{1}$-ness of $\partial \Omega$. The generator of the geodesic flow, $X$ is also $C^{1, \alpha}$, being tangent to a smooth foliation and being normalized with respect to the Hilbert metric. But if we reparameterize the flow, using a smooth Riemannian metric, the resulting vector field $Z$ is smooth, so it is for the induced flow. However $F$ is $C^{1, \alpha}$, hence $\beta$ is $C^{1, \alpha}$.

We have the following theorem about the change of time, due to Anosov and Sinai [2, 36].

Theorem 3.2. If $\phi$ is a $C^{1}$-Anosov flow and $\psi$ is obtained from $\phi$ by multiplying a positive $C^{1}$-function on the speed, then $\psi$ is again Anosov.

Remark 3.3. In our case, $\psi$ is a $C^{\infty}$-Anosov flow. This is the $C^{\infty}$ foliation by straight lines with $C^{\infty}$ parametrization.

Now using the result of Parry [36] we get,

$$
\mu_{S R B}^{\phi}=\beta \mu_{S R B}^{\psi} / \int_{H M} \beta d \mu_{S R B}^{\psi}
$$

and using a theorem of Abramov [1]

$$
h_{S R B}(\phi)=h_{S R B}(\psi) / \int_{H M} \beta d \mu_{S R B}^{\psi} .
$$

The continuity of the SRB entropy for $C^{\infty}$ Anosov flow is due to Contreras [16] (theorem B). 
Theorem 3.4. If $\lambda$ is a $C^{r}$-Anosov flow, there exists a neighborhood $U$ of $\lambda$ in $C^{r}$ topology such that the function $\psi \rightarrow h_{S R B}(\psi)$ is $C^{r-2}$.

To control the denominator let us recall that the SRB measure is the unique equilibrium state associated with the infinitesimal volume expansion which is here a smooth function. Since the scaling factor $\beta$ is $C^{1, \alpha}$, we may again invoke [16] (theorem $\mathrm{C}$ ) that we may specialize to SRB measures as follows

Theorem 3.5. If $\lambda$ is a $C^{r}$-Anosov flow, there exists a neighborhood $U$ of $\lambda$ in $C^{r}$ topology such that if $\mu_{\lambda}$ is its SRB measure then for any $0<\alpha<1$ small enough the map $\lambda \in U \rightarrow \mu_{\lambda} \in C^{\alpha}(M, \mathbb{R})^{*}$ is $C^{r-1}$.

Now we prove that the entropy of SRB along a smooth deformation varies continuously.

Theorem 3.6. Let $\rho_{t}: \pi_{1}(M) \rightarrow S L(n+1, \mathbb{R})$ be a smooth deformation of strictly convex projective structures. Then $t \rightarrow h_{S R B}\left(\rho_{t}\right)$ is a continuous map.

Proof. Since $\Omega_{t}=D_{t}(\widetilde{M})$ is continuously determined by $\rho_{t}$ as in Section 2.4, both $\Omega_{t}$ and $\partial \Omega_{t}$ depend continuously on $t$ with respect to the Hausdorff topology. Fix a $C^{\infty}$ Riemannian metric on $M$. Now since $\rho_{t}$ depends smoothly on $t$, the reparameterized geodesic flow $\psi_{t}$ depends smoothly on $t$. By Equation (9),

$$
\beta_{t}=F_{t}\left(d \pi Z_{t}\right)
$$

is continuous in $t$. Then, by Equation (11), and since $h\left(\psi_{t}\right)_{S R B}$ varies continuously by Theorem 3.4, it is enough to show that the denominator varies continuously.

Lemma 3.7. Let $\alpha$ be as in theorem 3.5. If $f_{i} \in C^{\alpha}(M, \mathbb{R})$ converges to $f \in C^{\alpha}(M, \mathbb{R})$ in $C^{0}$ topology and probability measures $\mu_{i}$ converge to $\mu$ in $C^{\alpha}(M, \mathbb{R})^{*}$, then

$$
\int_{M} f_{i} d \mu_{i} \rightarrow \int_{M} f d \mu
$$

Proof.

$$
\begin{gathered}
\left|\int f_{i} d \mu_{i}-\int f d \mu\right| \leq\left|\int f_{i} d \mu_{i}-\int f d \mu_{i}\right|+\left|\int f d \mu_{i}-\int f d \mu\right| \\
\leq\left|f_{i}-f\right|_{\infty}+\left|\int f d\left(\mu_{i}-\mu\right)\right| .
\end{gathered}
$$

The first term goes to zero since $f_{i} \rightarrow f$ in $C^{0}$ topology and the second term goes to zero since $\mu_{i} \rightarrow \mu$ in $C^{\alpha}(M, \mathbb{R})^{*}$. 
Corollary 3.8. Along the smooth deformation of convex real projective structures, the sum $\eta$ of parallel Lyapunov exponents varies continuously.

Proof. Since the geodesic flow $\phi$ is ergodic with respect to the SRB probability measure $\mu$, and the measurable function $\chi^{+}$is $\phi$-invariant, $\chi^{+}$is almost constant. Furthermore, by the property of the SRB measure, $h_{\mu}(\phi)=\int \chi^{+} d \mu$, the entropy $h_{\mu}(\phi)$ must be equal to $\chi^{+}$. Since the entropy varies continuously along the smooth deformation, $\chi^{+}$varies continuously along the smooth deformation. The claim follows from Equation (5).

In particular, we get

Corollary 3.9. Along the smooth deformation of the convex structure, the harmonic mean $\alpha$ varies continuously.

Proof. This follows from Eqn (7) and the above corollary 3.8 .

Now we give a proof for Corollary 1.2

Proof. The continuity follows from the main Theorem 1.1 and the other claims follow from results in [17] and equalities $\chi_{i}^{+}=1+\eta_{i}, \chi_{i}^{-}=$ $-1+\eta_{i}$. (4) Let $\omega$ be a volume form. Note that $T H M=E^{s} \oplus$ $E^{u} \oplus \mathbb{R} X$ where $X$ is the vector field generating the geodesic flow $\phi^{s}$ of the Hilbert metric. Choose a Riemannian metric $g$ so that for each point $w \in T_{w} H M, e_{1}(w), \cdots, e_{n-1}(w)$ are bases of $E^{u}(w)$ consisting of unit vectors, and $b_{1}(w), \cdots, b_{n-1}(w)$ bases of $E^{s}(w)$ consisting of unit vectors. Since $M$ is compact and $\omega$ is a continuous volume form,

$$
\omega\left(e_{1}, \cdots, e_{n-1}, b_{1}, \cdots, b_{n-1}, X\right)<N
$$

on $H M$ for some positive $N$. Since the flow $\phi^{s}$ preserves the splitting of $E^{u}, E^{s}$,

$$
d \phi^{s}\left(e_{i}(w)\right) \in E^{u}\left(\phi^{s}(w)\right), d \phi^{s}\left(b_{i}(w)\right) \in E^{s}\left(\phi^{s}(w)\right), d \phi^{s}(X)=X .
$$

Then

$$
\begin{gathered}
\lim _{s \rightarrow \infty} \frac{1}{s} \log \left(\frac{d\left(\phi^{s}\right)^{*}(\omega)\left(e_{i}, b_{i}, X\right)}{\omega\left(e_{i}, b_{i}, X\right)}\right)= \\
\lim _{s \rightarrow \infty} \frac{1}{s} \log \left(\frac{\omega\left(d \phi^{s}\left(e_{i}, b_{i}, X\right)\right)}{\omega\left(e_{i}, b_{i}, X\right)}\right)=\lim _{s \rightarrow \infty} \frac{1}{s} \log \left(\omega\left(d \phi^{s}\left(e_{i}, b_{i}, X\right)\right)\right) \\
=\lim _{s \rightarrow \infty} \frac{1}{s} \log \left(\left|\operatorname{det} d \phi^{s}\right|\right)=\sum \chi_{i} \operatorname{dim} E_{i}=2 \eta
\end{gathered}
$$


The last equality follows from the fact that $\chi_{i}^{+}=1+\eta_{i}, \chi_{i}^{-}=-1+\eta_{i}$ in Equation (4) and the second to the last equality follows from Equation (2).

Proof for Corollary 1.3 .

Proof. First note that the equality $\chi^{+} \alpha=2(n-1)$ follows from Equation (11). By ergodicity of the SRB measure, there exists a flow invariant set $W_{S R B} \subset H M$ of full measure with respect to the SRB measure on which the sum $\chi^{+}$of positive Lyapunov exponents is constant and coincide with the value of the SRB entropy. See the proof of Corollary 3.8. The set $W_{S R B} \subset H M$ being of full SRB measure, for almost all unstable leaf with respect to the invariant transverse measure induced by the SRB measure, almost every trajectory with respect to the conditional measure on that unstable leaf is regular and the sum of exponents for the parallel transport is $\eta$. We know that the conditional measure on unstable leaves of the SRB measure is absolutely continuous with respect to the Lebesgue measure, therefore for each such unstable leaf $L^{u}(x,[v])$ there exists a subset of full Lebesgue measure $S_{L^{u}(x,[v])} \subset \partial \Omega$ such that any boundary point $\xi \in S_{L^{u}(x,[v])}$ is approximately regular with exponent

$$
\alpha_{S R B}=\alpha(\xi)=\frac{2(n-1)}{n-1+\eta} \geq 2
$$

from Eqn (7).

Furthermore, this shows that the sum of exponents $\eta$ for the parallel transport is, on $W_{S R B}$, a non-positive number $\eta \leq 0$ such that $h_{S R B}=$ $n-1+\eta$ according to Eqn (5) and Crampon's inequality $h_{S R B} \leq n-1$ for any invariant measure [18]. We observe that $\eta=0$ is equivalent to being Riemannian hyperbolic [18], again equivalent to $\alpha_{S R B}=2$. The continuity of $\alpha_{S R B}: \mathcal{P}_{n}(M) \rightarrow \mathbb{R}$ follows from Corollary 3.9.

In the hyperbolic case every orbit of the flow is regular. We will observe that this is no longer true when $\eta<0$, which is what we will assume in the sequel. Again, the equality $\alpha_{S R B}=2$ is achieved only in the hyperbolic case.

Remark: We can go further and observe some strange effect that will shed some light on the transversal measure of the SRB measure. Such an unstable leaf is associated to a point $\xi^{-}$on the boundary such that all the geodesics under consideration emanate from that point at $-\infty$. However, we know that any regular geodesic is also regular by reversing time and then all the reversed geodesics have opposite parallel transport exponent. Then the approximately regular exponent at $\xi^{-}$ 
is $\alpha\left(\xi^{-}\right)=\frac{2}{1-\eta} \leq 2$. Such points have a measure 1 with respect to the transverse SRB measure which is therefore orthogonal to Lebesgue as soon as it is not hyperbolic. To sum up, there exists on the boundary a set of full Lebesgue measure of points on which the convex is "flatter" $(\alpha>2)$ than a conic and a zero Lebesgue measure of points where the convex is "sharper" $(\alpha<2)$ than a conic.

Remark: A funny remark is that when $\eta<0$ there is no triangle with regular geodesics but there are many even sided regular polygons with regular geodesics.

\section{REFERENCES}

[1] L. Abramov, On the entropy of a flow, Am. Math. Soc. Transl. 49 (1966), 167-170.

[2] D. Anosov and Y. Sinai, Some smooth ergodic systems, Russ. Math. Survey. 22 (1967), 103-167.

[3] L. Barreira and Y. B. Pesin, Nonuniform hyperbolicity, Cambridge university Press, 2007.

[4] Y. Benoist, Convexes divisibles I, Algebraic groups and arithmetic, Tata Inst. Fund. Res. Stud. Math, 17 (2004), 339-374.

[5] Y. Benoist and D. Hulin, Cubic differentials and finite volume convex projective surfaces, JGD, 98 (2014), 1-19.

[6] J-P Benzécri, Sur les variétés localement affines et localement projectives, Bull. Soc. Math. France, 88 (1960), 229-332.

[7] N. Bergeron and T. Gelander, A note on local rigidity, Geom. Dedicata, 107 (2004), 111-131.

[8] F. Bonahon and G. Dreyer, Parameterizing Hitchin components, Duke Math. J. 163 (2014), no. 15, 2935-2975.

[9] F. Bonahon and I. Kim, The Goldman and Fock-Goncharov coordinates for convex projective structures on surfaces, Geom. Dedicata, 192 (2018), 43-55.

[10] R. Bowen, Periodic orbits of hyperbolic flows, Amer. J. Math., 94 (1972).

[11] R. Bowen and D. Ruelle, The ergodic theory of axiom A flows, Inventiones Math., 29 (1975),181-202.

[12] O. Butterley and C. Liverani, Smooth Anosov flows: Correlation spectra and stability, J. Mod. Dyn. 1 (2007), 301-322.

[13] E. Calabi, Complete affine hyperspheres I., In Symposia Mathematica, Vol. X, pages 19-38, 1972.

[14] S. Cheng and S. Yau, On the regularity of the monge-ampére equation $\operatorname{det}\left(\partial^{2} u / \partial x_{i} \partial s x_{u}\right)=F(x, u)$, Comm. Pure Appl. Math., 30 (1977), 41-68.

[15] S. Choi and W. Goldman, Convex real projective structures on closed surfaces are closed, Proc. Amer. Math. Soc. 118 (1993), no. 2, 657-661.

[16] G. Contreras, Regularity of topological and metric entropy of hyperbolic flows, Math. Z, 210 (1992), 97-111,

[17] M. Crampon, Dynamics and entropies of Hilbert metrics, Thèse, Université de Strasbourg, 2011.

[18] M. Crampon, Entropies of strictly convex projective manifolds, J. Mod. Dyn. 3 (2009), 511-547. 
[19] M. Crampon, Lyapunov exponents in Hilbert geometry, Ergodic theory Dynam. System 34 (2014), 501-533.

[20] M. Crampon, The boundary of a divisible convex set, Publ. Mat. Urug. 14 (2013), 105-119.

[21] V. Fock and A. Goncharov, Moduli spaces of convex projective structures on surfaces, Adv. Math. 208 (2007), no. 1, 249-273.

[22] P. Foulon, Géométrie des équations différentielle du second ordre, Ann. Inst. Henri Poincaré, 45 (1986), 1-28.

[23] P. Foulon, Estimation de l'entropie des systèmes lagrangiens sans points conjugués, Ann. Inst. Henri Poincaré Phys. Théor., 57 (2) (1992), 117-146. With an appendix, "About Finsler geometry".

[24] W. Goldman, Convex real projective structures on compact surfaces, JDG, 31 (1990), 791-845.

[25] N. Hitchin, Lie groups and Teichmüller space, Topology, 31(1992), 449-473.

[26] I. Kim and G. Zhang, Kähler metric on the space of convex real projective structures on surface, JDG, 106 (2017), 127-137.

[27] S. Kobayashi, Foundations of Differential Geometry, Volume 1, Interscience Publishers, 1963.

[28] N. Kuiper, On convex locally projective spaces, Convegno Internazionale di Geometria Differentiale, Cremonese (1954), 200-213.

[29] F. Labourie, Flat projective structures on surfaces and cubic holomorphic differentials, Pure Appl. Math. Q, 3 (2007), 1057-1099.

[30] F. Labourie, Anosov flows, surface groups and curves in projective space, Invent. Math. 165 (2006), 51-114.

[31] F. Ledrappier, Structure au bord des variétés à courbure négative, Séminaire de théorie spectrale et géométrie, 71, 1994-1995.

[32] F. Ledrappier and L.-S. Young, The metric entropy of diffeomorphism, Ann. of Math., 122 (1985), 509-574.

[33] J. Loftin, Affine spheres and convex $R P^{n}$ manifolds, American Journal of Math. 123 (2) (2001), 255-274.

[34] J. Loftin, The compactification of the Moduli space of convex $R P^{2}$-surfaces I, JDG 68 (2004), 223-276.

[35] V.I. Oseledec, A multiplicative ergodic theorem, Trans. Moscow Math. Soc., 19 (1968), 197-231.

[36] W. Parry, Synchronisation of Canonical measures for hyperbolic attractors, Comm. Math. Phys. 106 (1986), 267-275.

[37] D. Ruelle, An inequality for the entropy of differentiable maps, Bol. Soc. Brasil. Mat. 9 (1978), 83-87.

[38] D. Ruelle, Differentiation of SRB states for hyperbolic flow, Ergodic Theory Dynam. Systems 28 (2008), 613-631.

Patrick Foulon

Aix-Marseille Université, CNRS, Société Mathématique de France, CIRM (Centre International de Rencontres Mathématiques), Marseille, France. UMR 822, 163 avenue de Luminy

13288 Marseille cedex 9, France 
foulon@cirm-math.fr

Inkang Kim

School of Mathematics

KIAS, Heogiro 85, Dongdaemen-gu

Seoul, 02455, Korea

inkang@kias.re.kr 